\title{
CASTELNUOVO REGULARITY AND GRADED RINGS ASSOCIATED TO AN IDEAL
}

\author{
BERNARD JOHNSTON AND DANIEL KATZ
}

(Communicated by Wolmer V. Vasconcelos)

\begin{abstract}
We compare the Castelnuovo regularity defined with respect to different homogeneous ideals in a graded ring and use the result we obtain to prove a generalized Goto-Shimoda theorem for ideals of positive height in a Cohen-Macaulay local ring.
\end{abstract}

\section{INTRODUCTION}

Let $(R, \mathfrak{m})$ be a Cohen-Macaulay local ring and $I \subseteq R$ an ideal. A number of papers in the past ten years or so have studied the transfer of the CohenMacaulay property of $R$ to various graded rings associated to $I$, with particular attention being paid to $\mathscr{G}=\mathscr{G}(I)$ and $\mathscr{R}=\mathscr{R}(I)$ - the associated graded ring and the Rees ring of $R$ with respect to $I$. In [H], Huneke showed that $\mathscr{G}$ is Cohen-Macaulay whenever $\mathscr{R}$ is Cohen-Macaulay (and the ideal has positive height) and pointed out that the converse need not hold. Since then, numerous authors have studied additional conditions required for $\mathscr{R}$ to be CohenMacaulay when $\mathscr{G}$ is Cohen-Macaulay. One of the most important theorems to emerge from these endeavors is the so-called Goto-Shimoda theorem ([GS, Theorem 3.1]) which we now state.

Theorem (Goto-Shimoda). Let $(R, \mathfrak{m})$ be a d-dimensional Cohen-Macaulay local ring with infinite residue field and $I \subseteq R$ an m-primary ideal. Then $\mathscr{R}$ is Cohen-Macaulay if and only if $\mathscr{G}$ is Cohen-Macaulay and $J I^{d-1}=I^{d}$ for every minimal reduction $J$ of $I$.

It is fair to say that [GS] has provided the impetus for a large amount of research. Notable among subsequent endeavors is [GHO], where the GotoShimoda theorem was extended to equimultiple ideals (i.e., ideals whose height equals their analytic spread). The theorem in [GHO] reads exactly the same as the one above, only the assumption that $I$ is m-primary is replaced by the (more general) assumption that $I$ is equimultiple and $d=\operatorname{dim}(R)$ is replaced by $s=s(I)$, the analytic spread of $I$. Little progress was made on extending the

Received by the editors June 21, 1993.

1991 Mathematics Subject Classification. Primary 13A20, 13D45, $13 \mathrm{H} 10$.

The second author was partially supported by the General Research Fund at the University of Kansas. 
original result further, until the recent paper [GH]. There, using the concept of analytic deviation introduced by Huckaba and Huneke in [HH1] and [HH2], the authors extended the Goto-Shimoda theorem to ideals with analytic deviation one (i.e., height $(I)+1=s(I)$ ). Goto and Huckaba's theorem reads as follows:

Theorem (Goto-Huckaba). Let $(R, \mathfrak{m})$ be a Cohen-Macaulay local ring with infinite residue field and $I \subseteq R$ an ideal having positive height which is generically a complete intersection and has analytic deviation one. Let $s=s(I)$. Then $\mathscr{R}$ is Cohen-Macaulay if and only if $\mathscr{G}$ is Cohen-Macaulay and $J I^{s-1}=I^{s}$ for every minimal reduction $J$ of $I$.

In the meantime, a different approach to the question of when $\mathscr{R}$ is CohenMacaulay was taken by Trung and Ikeda in [TI]. Using graded local cohomology of $\mathscr{G}$ with respect to its homogeneous maximal ideal, they were able to characterize completely when $\mathscr{R}$ is Cohen-Macaulay for any ideal in any local ring. A particular case of their theorem, relevant to the discussion at hand, is the result below. In the statement of this result, we write $a_{d}(\mathfrak{M}, \mathscr{G})$ for the smallest integer $k$ such that $H_{\mathfrak{M}}^{d}(\mathscr{G})_{j}=0$, for all $j>k$, where $H_{\mathfrak{M}}^{d}(\mathscr{G})$ denotes the $d$ th local cohomology module of $\mathscr{G}$ with respect to its homogeneous maximal ideal $\mathfrak{M}$.

Theorem (Trung-Ikeda). Let $(R, \mathfrak{m})$ be a d-dimensional Cohen-Macaulay local ring and $I \subseteq R$ an ideal having positive height. Then $\mathscr{R}$ is Cohen-Macaulay if and only if $\mathscr{G}$ is Cohen-Macaulay and $a_{d}(\mathfrak{M}, \mathscr{G})<0$.

This result, aside from its intrinsic elegance, has been extremely useful. In fact, using it, one may readily recover the original Goto-Shimoda theorem and the version given in [GHO]. Moreover, the theorem of Trung-Ikeda also plays a crucial role in the main result of [GH]. At first blush, there may not seem to be a connection between the Goto-Shimoda theorem and the Trung-Ikeda theorem, but indeed there is. This connection is provided by the following result $[\mathrm{T}$, Proposition 3.2].

Proposition (Trung). Let $(R, \mathfrak{m})$ be a local ring with infinite residue field and $I \subseteq R$ an ideal. For any minimal reduction $J \subseteq I$, write $r_{J}(I)$ for the least integer $n$ such that $J I^{n}=I^{n+1}$ and let $s=s(I)$. Then

$$
a_{s}\left(\mathscr{G}_{+}, \mathscr{G}\right)+s \leq r_{J}(I) \leq \max \left\{a_{i}\left(\mathscr{G}_{+}, \mathscr{G}\right)+i \mid i=0, \ldots, s\right\} .
$$

In this proposition, $a_{i}\left(\mathscr{G}_{+}, \mathscr{G}\right)$ is the least integer $k_{i}$ such that $H_{\mathscr{G}_{+}}^{i}(\mathscr{G})_{j}=$ 0 for all $j>k_{i}$, where $H_{\mathscr{G}_{+}}^{i}(\mathscr{G})$ denotes the $i$ th local cohomology module of $\mathscr{G}$ with respect to $\mathscr{G}_{+}$. Using the proposition and the theorem of TrungIkeda, the Goto-Shimoda result is immediate and, using some fairly standard reduction techniques, the equimultiple case can be reduced to the m-primary case and so may also be recovered. (The interested reader is urged to read the proof in [GHO], where an extremely nice noncohomological argument is given.) The problem of deducing a general Goto-Shimoda theorem should now be apparent. The Trung-Ikeda theorem provides a means to deduce that $\mathscr{R}$ is Cohen-Macaulay based upon information concerning the local cohomology modules of $\mathscr{G}$ with respect to $\mathfrak{M}$, while in turn, the reduction number of $I$ is determined by information based upon the local cohomology modules of $\mathscr{G}$ with respect to $\mathscr{G}_{+}$. It is the goal of the present paper to establish a connection 
between these modules, in as much as we seek to relate the vanishing of the degree $j$ components of the module $H_{\mathfrak{M}}^{d}(\mathscr{G})$ with the vanishing of the degree $j$ components of the modules $H_{\mathscr{G}_{+}}^{i}(\mathscr{G})$. This is done in Proposition 2.1 below. Using this result we are able to prove the following generalization of the GotoShimoda theorem. In the statement of the-result, we let $\mathscr{A}(I)$ denote the necessarily finite set of primes $P$ containing $I$ for which $s\left(I_{P}\right)=\operatorname{dim}\left(R_{P}\right)$.

Theorem 2.3 (Generalized Goto-Shimoda). Let $(R, \mathfrak{m})$ be a Cohen-Macaulay local ring with infinite residue field and $I \subseteq R$ an ideal having positive height. Then the following statements are equivalent:

(i) $\mathscr{R}$ is Cohen-Macaulay.

(ii) $\mathscr{G}$ is Cohen-Macaulay and $\operatorname{rd}_{J_{P}}\left(I_{P}\right) \leq s\left(I_{P}\right)-1$, for all prime ideals $P$ containing $I$ and all minimal reductions $J_{P}$ of $I_{P}$.

(iii) $\mathscr{G}$ is Cohen-Macaulay and $\operatorname{rd}\left(I_{P}\right) \leq s\left(I_{P}\right)-1$, for all prime ideals $P \in \mathscr{A}(I)$.

Here we are using $\operatorname{rd}(I)$ to denote the reduction number of $I$, which is defined to be the least integer $\operatorname{rd}_{J}(I)$ such that $J \subseteq I$ is a minimal reduction. Note that if we let $s=s(I)$ and assume that $I$ is equimultiple or generically a complete intersection with analytic deviation one, then the condition $\operatorname{rd}\left(I_{P}\right) \leq$ $s\left(I_{P}\right)-1$ holds for all prime ideals $P$ containing $I$ if we assume $J I^{s-1}=I^{s}$ for every minimal reduction $J \subseteq I$, so we have recovered the previously mentioned extensions of the original Goto-Shimoda theorem.

We now briefly describe the notation and terminology used in this paper. Let $(R, \mathfrak{m})$ be a $d$-dimensional local ring with residue field $k$. For an ideal $I \subseteq R$, we consider $\mathscr{G}:=\mathscr{G}(I):=\bigoplus_{j \geq 0} I^{j} / I^{j+1}$, the associated graded ring of $R$ with respect to $I$ and $\mathscr{R}:=\mathscr{R}(I):=\bigoplus_{j \geq 0} I^{j}$, the Rees ring of $R$ with respect to $I$. Closely related is the fiber ring of $R$ with respect to $I$, $\mathscr{F}:=\mathscr{F}(I):=\bigoplus_{j \geq 0} I^{j} / \mathfrak{m} I^{j}$. Recall that $s(I)$, the analytic spread of $I$, is the Krull dimension of $\mathscr{F}$. Writing $\mathscr{A}(I)$ for the prime ideals containing $I$ for which $s\left(I_{P}\right)=\operatorname{dim}\left(R_{P}\right)$, it follows from [Mc, Chapter 4] that if $R$ is quasiunmixed (hence, in particular, if $R$ is Cohen-Macaulay), then $P \in \mathscr{A}(I)$ if and only if there exists a minimal prime $\mathfrak{P}$ of $I \mathscr{R}$ such that $\mathfrak{P} \cap R=P$. Therefore, $\mathscr{A}(I)$ is a finite set of prime ideals. An ideal $J \subseteq I$ is said to be a reduction of $I$ iff there exists a power $n$ such that $J I^{n}=I^{n+1}$. The reduction number of $I$ with respect to $J$, denoted $\operatorname{rd}_{J}(I)$, is the least power $n$ making $J$ a reduction of $I . J$ is called a minimal reduction iff $J$ contains no other reductions of $I$. The reduction number of $I$, denoted $\operatorname{rd}(I)$, is the least number $\operatorname{rd}_{J}(I)$, as $J$ ranges over the minimal reductions of $I$. It is a standard fact (q.v. [NR]) that the minimal number of generators of any minimal reduction of $I$ will always be $s(I)$ (assuming the residue field to be infinite).

We now briefly recall the concept of local cohomology. If $I$ is an ideal in the ring $R$ and $N$ is a finite $R$-module, the $i$ th local cohomology module of $N$ with respect to $I$, denoted $H_{I}^{i}(N)$, is defined via the formula

$$
H_{I}^{i}(N):=\underset{n}{\lim } \operatorname{Ext}_{R}^{i}\left(R / I^{n}, N\right) .
$$

It can also be obtained as the $i$ th cohomology of the Čech complex:

$$
C^{\cdot}: \quad 0 \rightarrow N \rightarrow N_{b_{1}} \oplus \cdots \oplus N_{b_{k}} \rightarrow \cdots \rightarrow N_{b_{1} \cdots b_{k}} \rightarrow 0,
$$


where the modules are the direct sums of the various localizations, taken none at a time, one a time, two at a time, etc., and where the maps are just the sums of the canonical maps into the localizations (with appropriate minus signs), and where $\left(b_{1}, \ldots, b_{k}\right)=J$ is any ideal having the same radical as $I$. Note that $H_{I}^{0}(N)$ can be identified with $\bigcup_{n}\left(\begin{array}{lll}0 & :_{N} & I^{n}\end{array}\right)$, the set of elements in $N$ annihilated by some power of $I$. In the case that $R$ and $N$ are graded and $I$ is homogeneous, one may take the elements $b_{1}, \ldots, b_{k}$ occurring in the complex $C^{*}$ to be a set of homogeneous generators for any ideal $J$ having the same radical as $I$. Consequently, the modules in the complex $C^{\cdot}$ and hence the homology modules of the complex retain a natural graded structure, and it is this which is generally referred to as graded local cohomology. See [HIO, Chapter VII] for more information.

We should also mention that during the preparation of this manuscript we were informed that the main result of this paper, Theorem 2.3, has been obtained independently by Aberbach-Huneke-Trung using different techniques (see [AHT]). Moreover, very closely related results have been obtained via yet another set of techniques by Simis-Ulrich-Vasconcelos (see [SUV]). Both papers should be forthcoming.

\section{Comparing Castelnuovo Regularities}

We begin by considering the vanishing in certain degrees of local cohomology modules defined with respect to different homogeneous ideals. In the proposition below we fix a $d$-dimensional graded Noetherian ring $\mathbf{A}=\bigoplus_{j \geq 0} A_{j}$. We write $B=\mathbf{A}_{0}$ for the degree zero component of $\mathbf{A}$ and assume that $\bar{B}$ is a local ring with maximal ideal $\mathfrak{m}$. For $p \in \operatorname{Spec}(B)$ write $\mathbf{A}_{p}$ for $\mathbf{A}$ localized at the multiplicatively closed set $B \backslash p$, and set $\mathfrak{P}=\left(p, \mathbf{A}_{p_{+}}\right) \mathbf{A}_{p}$ and $d(p)=\operatorname{dim}\left(\mathbf{A}_{p}\right)$. For a homogeneous ideal $\mathfrak{J} \subseteq \mathbf{A}$, we will write $a_{i}(\mathfrak{J}, \mathbf{A})$ for the least integer $k_{i}$ (including $-\infty$ ) such that $H_{\mathfrak{J}}^{i}(\mathbf{A})_{j}=0$, for all $j>k_{i}$. When $\mathfrak{J}=\mathbf{A}_{+}$, $\max \left\{a_{i}\left(\mathbf{A}_{+}, \mathbf{A}\right)+i \mid 0 \leq i \leq d\right\}$ is customarily called the Castelnuovo regularity of $\mathbf{A}$. By abuse of terminology, we shall call $\max \left\{a_{i}(\mathfrak{J}, \mathbf{A})+i \mid 0 \leq i \leq d\right\}$ the Castelnuovo regularity of $\mathbf{A}$ with respect to $\mathfrak{J}$. As mentioned above, we are interested in relating the Castelnuovo regularity of the associated graded ring of an ideal with respect to its homogeneous maximal ideal (as used in the Trung-Ikeda theorem) to the Castelnuovo regularity with respect to its positive part (as used in Trung's proposition concerning reduction number). Toward this end we offer the following proposition.

Proposition 2.1. Let A be a graded Noetherian ring over a local ring $B$. Then:

(i) Let $r$ be an integer such that $H_{\mathfrak{P}}^{i}\left(\mathbf{A}_{p}\right)_{j}=0$, for all $j \geq r, p \in \operatorname{Spec}(B)$, and all $i, \quad 0 \leq i \leq d(p)$. Then for any homogeneous ideal $\mathfrak{J}$ which contains $\mathbf{A}_{+}, H_{\mathfrak{J}}(\mathbf{A})_{j}=0$ for all $j \geq r$ and $0 \leq i \leq d$.

(ii) Conversely, suppose that $s$ is an integer satisfying $H_{\mathbf{A}_{+}}^{i}(\mathbf{A})_{j}=0$ for all $j \geq s$ and $0 \leq i \leq d$. Then, for all $p \in \operatorname{Spec}(B)$ and $0 \leq i \leq d(p), H_{\mathfrak{P}}^{i}\left(\mathbf{A}_{p}\right)_{j}=$ 0 for all $j \geq s$.

(iii) Let $t$ be an integer such that $H_{\mathbf{A}_{p_{+}}}^{d(p)}\left(\mathbf{A}_{p}\right)_{j}=0$, for all $j \geq t$ and $p \in$ $\operatorname{Spec}(B)$. Then for any homogeneous ideal $\mathfrak{J}$ which contains $\mathbf{A}_{+}, H_{\mathfrak{J}}^{d}(\mathbf{A})_{j}=0$, for all $j \geq t$. 
Before turning to the proof of the proposition, we make a couple of remarks concerning (graded) local cohomology.

Remarks. (a) If $R$ is a (not necessarily graded) Noetherian ring, $x \in R$, and $I \subseteq R$ is an ideal, then according to [B, (3.9) Lemma], for any $R$-module $M$ there is a long exact sequence

$$
\begin{aligned}
& 0 \rightarrow H_{(I, x)}^{0}(M) \rightarrow H_{I}^{0}(M) \rightarrow H_{I_{x}}^{0}\left(M_{x}\right) \rightarrow H_{(I, x)}^{1}(M) \rightarrow \\
& \quad \ldots \rightarrow H_{(I, x)}^{i}(M) \rightarrow H_{I}^{i}(M) \rightarrow H_{I_{x}}^{i}\left(M_{x}\right) \rightarrow H_{(I, x)}^{i+1}(M) \rightarrow \cdots .
\end{aligned}
$$

If $R$ and $M$ are graded and $I$ and $x$ are homogeneous, then the maps in this sequence are degree-preserving.

(b) If $S \subseteq R$ is a multiplicatively-closed set then $H_{I}^{i}(M)_{S} \cong H_{I R_{S}}^{i}\left(M_{S}\right)$, i.e., local cohomology commutes with localization. If $R$ and $M$ are graded, $I$ is homogeneous, and $S \subseteq R_{0}$, then $\left[H_{I}^{i}(M)_{j}\right]_{S} \cong\left[H_{I R_{S}}^{i}\left(M_{S}\right)\right]_{j}$ as $\left(R_{0}\right)_{S}$-modules.

(c) It follows from (b) that if $R$ and $M$ are graded, $x \in R_{0}$, and $I$ is homogeneous, then $H_{I_{x}}^{i}\left(M_{x}\right)_{j}=0$ if and only if for all $p \in \operatorname{Spec}\left(R_{0}\right)_{x}$, $\left[H_{I R_{p}}^{i}\left(M_{p}\right)\right]_{j}=0$.

Proof of Proposition 2.1. To prove (i), suppose $H_{\mathfrak{P}}^{i}\left(\mathbf{A}_{p}\right)_{j}=0$ for all $j \geq r$, all $p \in \operatorname{Spec}(B)$, and all $i, 0 \leq i \leq d(p)$. In order to prove $H_{\mathfrak{J}}^{i}(\mathbf{A})_{j}=0$ for all $j \geq r$ and $0 \leq i \leq d$, we proceed by induction on $\operatorname{dim}(\mathbf{A} / \mathfrak{J})$. If $\operatorname{dim}(\mathbf{A} / \mathfrak{J})=0, \mathfrak{J}$ has radical equal to the homogeneous maximal ideal of $\mathbf{A}$, and hence the same local cohomology modules, so there is nothing to prove. Now suppose $\operatorname{dim}(\mathbf{A} / \mathfrak{J})>0$. Since $\mathbf{A}_{+} \subseteq \mathfrak{J}$, we may find a homogeneous element $x \in B$ of degree zero such that $\operatorname{dim}(\mathbf{A} /(\mathfrak{J}, x))<\operatorname{dim}(\mathbf{A} / \mathfrak{J})$. Consider the "degree $j$ level" of the Brodmann exact sequence:

$$
\cdots \rightarrow H_{\mathfrak{J} \mathbf{A}_{x}}^{i-1}\left(\mathbf{A}_{x}\right)_{j} \rightarrow H_{(\mathfrak{J}, x)}^{i}(\mathbf{A})_{j} \rightarrow H_{\mathfrak{J}}^{i}(\mathbf{A})_{j} \rightarrow H_{\mathfrak{J} \mathbf{A}_{x}}^{i}\left(\mathbf{A}_{x}\right)_{j} \rightarrow \cdots
$$

We claim that $H_{\mathfrak{J} \mathbf{A}_{x}}^{i}\left(\mathbf{A}_{x}\right)_{j}=0$. To see this, by the remarks above it suffices to show that $\left[H_{\mathfrak{J A}_{p}}^{i}\left(\mathbf{A}_{p}\right)\right]_{j}=0$ for all $p \in \operatorname{Spec}\left(B_{x}\right)$. But in the ring $\mathbf{A}_{p}$, the dimension of $\mathbf{A}_{p} / \mathfrak{J}_{p}$ has dropped, so this module is zero by induction on the dimension of $\mathbf{A} / \mathfrak{J}$. On the other hand, we also have that $H_{(\mathfrak{J}, x)}^{i}(\mathbf{A})_{j}=0$ by our induction hypothesis. By the Brodmann exact sequence, $H_{\mathfrak{J}}^{i}(\mathbf{A})_{j}=0$, as desired.

For the second part, we suppose that for all $i \geq 0$ and $j \geq s, H_{\mathbf{A}_{+}}^{i}(\mathbf{A})_{j}=0$, and we seek to prove that for all $p \in \operatorname{Spec}(B), i \geq 0$, and $j \geq s, H_{\mathfrak{P}}^{i}\left(\mathbf{A}_{p}\right)_{j}=0$. This time we may proceed by induction on $\operatorname{dim}(B)=\operatorname{dim}\left(\mathbf{A}_{0}\right)$. If $\operatorname{dim}(B)=0$, there is nothing to prove. Suppose $\operatorname{dim}(B)>0$. By localizing at $p \in \operatorname{Spec}(B)$ and applying our induction hypothesis, we see it is enough to consider the case that $p=\mathfrak{m}$ is the maximal ideal of $B$. Now, let $\mathfrak{J}$ be maximal among the homogeneous ideals of $\mathbf{A}$ containing $\mathbf{A}_{+}$such that $H_{\mathfrak{J}}^{i}(\mathbf{A})_{j}=0$, for all $i$ between 0 and $d$ and all $j \geq s$. We claim $\mathfrak{J}=\mathfrak{M}$, the homogeneous maximal ideal of $\mathbf{A}$. If not, then there exist $x \in B, 0 \leq i \leq d$, and $j \geq s$ such that $H_{(\mathfrak{J}, x)}^{i}(\mathbf{A})_{j} \neq 0$. However, $H_{\mathfrak{J} \mathbf{A}_{x}}^{i-1}\left(\mathbf{A}_{x}\right)_{j}$ is locally zero on $\operatorname{Spec}\left(B_{x}\right)$ by the induction on $\operatorname{dim} B$, and hence zero, so we conclude from Brodmann's exact sequence that $H_{(\mathfrak{J}, x)}^{i}(\mathbf{A})_{j}=0$, a contradiction.

Finally, to prove (iii), we again induct on $\operatorname{dim}(B)$. If $\operatorname{dim}(B)=0$, there is nothing to prove. Suppose $\operatorname{dim}(B)>0$. Then $d=\operatorname{dim}(\mathbf{A}) \geq 1$. Let 
$\mathbf{A}_{+} \subseteq \mathfrak{J}$, with $\mathfrak{J}$ homogeneous. Choose $\mathfrak{J}^{\prime}$ homogeneous, $\mathbf{A}_{+} \subseteq \mathfrak{J}^{\prime} \subseteq \mathfrak{J}$, and $\mathfrak{J}^{\prime}$ maximal with respect to the property that $H_{\mathfrak{J}^{\prime}}^{d}(\mathbf{A})_{j}=0$, for all $j \geq t$. If $\mathfrak{J}^{\prime} \neq \mathfrak{J}$, we may find $x \in \mathfrak{J}_{0}$ such that $\left(\mathfrak{J}^{\prime}, x\right)$ properly contains $\mathfrak{J}^{\prime}$ and such that $H_{\left(\mathfrak{J}^{\prime}, x\right)}^{d}(\mathbf{A})_{j} \neq 0$, for some $j \geq t$. Consider the end of the Brodmann sequence on the $j$ th level:

$$
\cdots \rightarrow H_{\mathfrak{J}^{\prime} \mathbf{A}_{x}}^{d-1}\left(\mathbf{A}_{x}\right)_{j} \rightarrow H_{\left(\mathfrak{J}^{\prime}, x\right)}^{d}(\mathbf{A})_{j} \rightarrow H_{\mathfrak{J}^{\prime}}^{d}(\mathbf{A})_{j} \rightarrow \cdots .
$$

Notice that $\left(H_{\mathfrak{J}^{\prime} \mathbf{A}_{x}}^{d-1}\left(\mathbf{A}_{x}\right)_{j}\right)_{p} \cong\left[H_{\mathfrak{J}^{\prime} \mathbf{A}_{p}}^{d-1}\left(\mathbf{A}_{p}\right)\right]_{j}$, for all $p \in \operatorname{Spec}\left(B_{x}\right)$, by the remarks above. For any such $p, d(p) \leq d-1$, so $\left[H_{\mathfrak{J}^{\prime} \mathbf{A}_{p}}^{d-1}\left(\mathbf{A}_{p}\right)\right]_{j}=0$ (either by induction on $\operatorname{dim}(B)$ or dimension-theoretic considerations). Thus $H_{\mathfrak{J}^{\prime} \mathbf{A}_{x}}^{d-1}\left(\mathbf{A}_{x}\right)_{j}=0$. From the exact sequence above, $H_{\left(\mathfrak{J}^{\prime}, x\right)}^{d}(\mathbf{A})_{j}=0$, contradiction.

Corollary 2.2. Let the notation be as in Proposition 2.1. Let $d=\operatorname{dim}(\mathbf{A})$, and suppose that for some integer $r, H_{\mathfrak{P}}^{i}\left(\mathbf{A}_{p}\right)_{j}=0$ for all $j \geq r$, all $p \in \operatorname{Spec}(B)$, and all $0 \leq i \leq d(p)$. Let $\mathfrak{J}$ be any homogeneous ideal containing $\mathbf{A}_{+}$. Then the Castelnuovo regularity of $\mathbf{A}$ with respect to $\mathfrak{J}$ is less than $d+r$.

Proof. Immediate from the definition of Castelnuovo regularity and Proposition 2.1.

We are now ready for our theorem.

Theorem 2.3 (Generalized Goto-Shimoda). Let $(R, \mathfrak{m})$ be a Cohen-Macaulay local ring with infinite residue field and $I \subseteq R$ an ideal having positive height. The following statements are equivalent:

(i) $\mathscr{R}$ is Cohen-Macaulay.

(ii) $\mathscr{G}$ is Cohen-Macaulay and $\operatorname{rd}_{J_{P}}\left(I_{P}\right) \leq s\left(I_{P}\right)-1$, for all prime ideals $P$ containing $I$ and all minimal reductions $J_{P}$ of $I_{P}$.

(iii) $\mathscr{G}$ is Cohen-Macaulay and $\operatorname{rd}\left(I_{P}\right) \leq s\left(I_{P}\right)-1$, for all prime ideals $P \in \mathscr{A}(I)$.

Proof. Suppose that $\mathscr{R}$ is Cohen-Macaulay. By Huneke's result [H, Proposition 1.1] $\mathscr{G}$ is Cohen-Macaulay. We want to show $\operatorname{rd}_{J_{P}}\left(I_{P}\right) \leq s\left(I_{P}\right)-1$, for all prime ideals $P$ containing $I$ and all minimal reductions $J_{P}$ of $I_{P}$. For this we may clearly localize at any $P$ containing $I$, assume $P=\mathfrak{m}$, and show $\operatorname{rd}_{J}(I) \leq s(I)-1$ for any minimal reduction $J$. Starting anew, we let $P \in \operatorname{Spec}(R)$ be any prime containing $I$ and set $d(P)=\operatorname{dim}\left(R_{P}\right)$. Write $\mathfrak{P}$ for the homogeneous maximal ideal of $\mathscr{G}_{P}$. Since $\mathscr{R}_{P}$ is also CohenMacaulay, $a_{d(P)}\left(\mathfrak{P}, \mathscr{G}_{P}\right)<0$ (by the theorem of Trung-Ikeda). Since $\mathscr{G}_{P}$ is Cohen-Macaulay, this is the only "a-invariant" of $\mathscr{G}_{P}$ to worry about, so we conclude from Proposition 2.1(i) (by taking $\mathscr{G}=\mathbf{A}, \mathscr{G}_{+}=\mathfrak{J}, p=P / I$, and $r=0)$ that $H_{\mathscr{G}_{+}}^{i}(\mathscr{G})_{j}=0$ for all $j \geq 0$ and $0 \leq i \leq d$. Moreover, since $\mathscr{G}_{+}$ may be generated up to radical by $s(I)$ elements (namely, the images in $I / I^{2}$ of a minimal reduction for $I$ ), it follows that $H_{\mathscr{G}_{+}}^{i}(\mathscr{G})=0$ for $i>s(I)$. Hence the Castelnuovo regularity of $\mathscr{G}$ with respect to $\mathscr{G}_{+}$is less than or equal to $s(I)-1$, and the required bound on the reduction number $r_{J}(I)$ now follows immediately from Trung's proposition [T, Proposition 3.2]. Thus (i) implies (ii). Clearly (ii) implies (iii). 
To see that (iii) implies (i), suppose that $\mathscr{G}$ is Cohen-Macaulay and $\operatorname{rd}\left(I_{P}\right) \leq$ $s\left(I_{P}\right)-1$, for all $P \in \mathscr{A}(I)$. By the Trung-Ikeda theorem, we need to show that $a_{d}(\mathfrak{M}, \mathscr{G})<0$. By taking $\mathbf{A}=\mathscr{G}$ and $\mathfrak{J}=\mathfrak{M}$ in the third part of Proposition 2.1, we see that it is enough to prove that $H_{\mathscr{G}_{P_{+}}}^{d(P)}\left(\mathscr{G}_{P}\right)_{j}=0$, for all $j \geq 0$ and $P \in \operatorname{Spec}(R)$ containing $I$. If $P \notin \mathscr{A}(I)$, then $s\left(I_{P}\right)<\operatorname{dim}\left(R_{P}\right)=$ $d(P)$, so $H_{\mathscr{G}_{P_{+}}}^{d(P)}\left(\mathscr{G}_{P}\right)=0$. If $P \in \mathscr{A}(I)$, then $\operatorname{rd}\left(I_{P}\right) \leq s\left(I_{P}\right)-1=d(P)-1$, so $H_{\mathscr{G}_{P_{+}}}^{d(P)}\left(\mathscr{G}_{P}\right)_{j}=0$, for all $j \geq 0$ (by Trung's proposition), and the proof is complete.

Corollary 2.4 [GHO], [GH]. Let $(R, \mathfrak{m})$ be a Cohen-Macaulay local ring with infinite residue field and let $I \subseteq R$ be an ideal having height at least one. Assume either that $I$ is equimultiple or that $I$ is generically a complete intersection with analytic deviation one. Let $s=s(I)$. Then $\mathscr{R}$ is Cohen-Macaulay if and only if $\mathscr{G}$ is Cohen-Macaulay and $\operatorname{rd}_{J}(I) \leq s-1$ for every minimal reduction $J$ of $I$.

Proof. Immediate from Theorem 2.3 and the definitions.

\section{ACKNOWLEDGMENT}

In the original version of this paper the proof of Theorem 2.3 was based on the Mayer-Vietoris sequence for local cohomology, and we were confined to ideals having height at least two. We are grateful to the referee for a careful reading of the manuscript and for pointing out that by using Brodmann's exact sequence [B, (3.9) Lemma] instead, the argument works for the height one case as well.

\section{REFERENCES}

[AHT] I. Aberbach, C. Huneke, and N. V. Trung, Reduction numbers, Briançon-Skoda theorems and the depth of the Rees algebra, preprint.

[B] M. Brodmann, Einige Ergebnisse aus der lokalen Kohomologie Theorie und ihre Anwendung, Osnabrück. Schrift. Math. Reihe Math. Manuskr., Heft 5, Univ. Osnabrück, Osnabrück, 1983.

[GH] S. Goto and S. Huckaba, On graded rings associated to analytic deviation one ideals, Amer. J. Math. (to appear).

[GHO] U. Grothe, M. Hermann, and U. Orbanz, Graded rings associated to equimultiple ideals, Math. Z. 186 (1984), 531-556.

[GS] S. Goto and Y. Shimoda, On the Rees algebras of Cohen-Macaulay local rings, Lecture Notes in Pure and Appl. Math., vol. 68, Marcel Dekker, New York, 1979, pp. 201-231.

[HIO] M. Hermann, S. Ikeda, and U. Orbanz, Equimultiplicity and blowing-up, Springer-Verlag, Berlin, 1988.

[HH1] S. Huckaba and C. Huneke, Powers of ideals having small analytic deviation, Amer. J. Math. 114 (1992), 367-403.

[HH2] S. Huckaba and C. Huneke, Rees algebras of ideals having small analytic deviation, Trans. Amer. Math. Soc. 399 (1993), 373-402.

[H] C. Huneke, On the associated graded ring of an ideal, Illinois J. Math. 26 (1982), 212-137.

[Mc] S. McAdam, Asymptotic prime divisors, Lecture Notes in Math., vol. 1023, Springer, Berlin, 1983. 
[NR] D. G. Northcott and D. Rees, Reductions of ideals in local rings, Math. Proc. Cambridge Philos. Soc. 50 (1952), 145-158.

[SUV] A. Simis, B. Ulrich, and W. Vasconcelos, Cohen-Macaulay Rees algebras and degrees of polynomial relations, preprint.

[T] N. V. Trung, Reduction exponent and degree bound for the defining equations of graded rings, Proc. Amer. Math. Soc. 101 (1987), 229-236.

[TI] N. V. Trung and S. Ikeda, When is the Rees ring Cohen-Macaulay?, Comm. Algebra 17 (1989), 2839-2922.

Department of Mathematics, Florida Atlantic University, Boca Raton, Florida 33431

E-mail address: johnstonecse.fau.edu

Department of MAThematics, University of Kansas, LAWRence, Kansas 66045

E-mail address: dlketitania.math.ukans.edu 